\title{
A Piezoactuated Droplet-Dispensing Microfluidic Chip
}

\author{
Mohammed Jalal Ahamed, Sergey I. Gubarenko, Ridha Ben-Mrad, and Pierre Sullivan
}

\begin{abstract}
A microfluidic dispensing device that is capable of generating droplets with volumes varying between $1 \mathrm{~nL}$ and $50 \mathrm{pL}$ at an ejection frequency of up to $6 \mathrm{kHz}$ is presented. In this device, a piezoactuator pushes onto an elastic membrane via piston tips; the mechanical bending of the membrane generates a pressure pulse pushing droplets out. An analytical model was developed solving bending characteristics of a plate-actuated fluidic dispensing system and used to calculate the displaced volume. The model was extended to perform stress analysis to find the optimum piston tip radius by minimizing design stresses. The optimum piston tip radius was found to be $67 \%$ of the chamber radius. The actuation force estimated using the analytical model was then used as input to a finite element model of the dispenser. A detailed numerical analysis was then performed to model the fluid flow and droplet ejection process and to find critical geometric and operating parameters. Results from both models were used together to find the best design parameters. The device contains three layers, a silicon layer sandwiched between two polydimethylsiloxane (PDMS) polymer layers. Silicon dry etching, together with PDMS soft lithography, was used to fabricate the chip. PDMS oxygen plasma bonding is used to bond the layers. Prototypes developed were successfully tested to dispense same-sized droplets repeatedly without unwanted droplets. The design allows easy expansion and simultaneous dispensing of fluids.

[2009-0099]
\end{abstract}

Index Terms-Deoxyribonucleic acid, droplet, ejection characteristics, microdispenser, microfluidics, piezoactuator, plate deflection.

\section{INTRODUCTION}

D EOXYRIBONUCLEIC acid microarray technology is used to identify gene sequences or to determine gene expression. Microarray dispensing typically involves delivering small samples/analytes on a substrate or slide with volumes in the range of nanoliters to picoliters [1]. Printing uniform spots within a slide and from slide to slide is important in a microarray application [2]. Existing dispensers can be categorized into contact printing with pins or noncontact printing [3]. There is a growing interest in using noncontact printing technology in microarray and biomedical diagnostic applications. Noncontact dispensing devices usually employ mechanical actuation to eject droplets through small nozzles. Presently, noncontact sys-

Manuscript received April 21, 2009; revised September 9, 2009. First published December 15, 2009; current version published February 3, 2010. This work was supported in part by the Natural Sciences and Engineering Research Council of Canada, in part by Engineering Services Inc., and in part by the Canadian Microelectronics Corporation. Subject Editor S. Shoji.

The authors are with the Mechatronics and Microsystems Design Laboratory, Department of Mechanical and Industrial Engineering, University of Toronto, Toronto, ON M5S 3G8, Canada (e-mail: rbenmrad@mie.utoronto.ca).

Color versions of one or more of the figures in this paper are available online at http://ieeexplore.iee.org.

Digital Object Identifier 10.1109/JMEMS.2009.2036866 tems utilize mainly the following actuation techniques: thermal actuation [4]-[6], pneumatic actuation [7], [8], electrostatic actuation [9], [10], acoustic actuation [11], [12], and piezoactuation [13]-[20]. There is a need for high-accuracy and highdensity microdroplet generators and for an understanding of the mechanics of droplet generators [14], [21]-[25].

A dispensing head, to be used in microarray production, should be biocompatible, so the operating conditions do not damage the biological samples. Nakamura et al. [26] showed that both piezoelectric and electrostatic actuation are suitable for biological sample printing. A number of research groups have presented piezoactuated [13]-[17] and electrostaticactuated [10] microdispensers for microarray printing. The piezoactuated dispensing head presented in this paper can be fabricated using low-cost microfabrication processes.

A noncontact-type droplet dispenser using plate actuation is presented. The microdispenser design is presented in Section II, which uses mechanical bending of an elastic plate due to the deflection of a piezoactuator. An analytical model was used to predict the volume dispensed based on the plate physical properties. A detailed finite element analysis solving for the droplet ejection was utilized to select the best values for the various geometric and operating parameters of the dispenser. The modeling and analysis are all presented in Section III. Experimental assessment of the prototype developed is described in Section IV.

The models presented in this paper assist in selecting geometric and operating variables of plate-actuated dispensers. The dispenser design presented in this paper could also be useful in other applications apart from microarray production, including ink-jet printing, droplet combustion studies, microoptical component manufacturing, direct writing, and electrical component cooling.

\section{SYSTEM DESCRIPTION}

The microdroplet generator (Fig. 1) consists of a piezoactuator driving a dispensing microfluidic chip. The dispensing unit consists of three basic elements: a pressure (fluid) chamber, an inlet microchannel, and a nozzle. The microfluidic chip consists of a silicon layer, containing the pressure chamber and the inlet channel, which is sandwiched between two polydimethylsiloxane (PDMS) layers. The lower PDMS layer is the nozzle layer, and the upper PDMS layer is the flexible plate layer with the piston tips. The dispensing unit could be arranged in an array format to generate multiple droplets simultaneously.

The dispenser works when a piezoactuator and the piston tip push on the plate, ejecting a droplet out through the nozzle. 


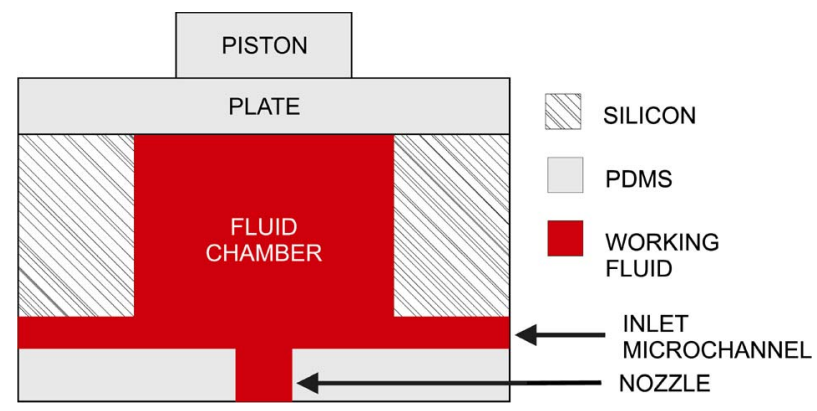

Fig. 1. Schematic of the dispensing device.

Initially, the reservoir is filled with liquid by an external syringe pump. The nozzle allows air to exit from the device during fill. After droplet ejection, the plate returns to its original position, drawing liquid in. To ensure proper refilling and to prevent backflow from the chamber during actuation, a small overpressure inside the microchannel and the pressure chamber is maintained with a pump at the inlet.

\section{Design Optimization}

The maximum plate deflection and bending line determine the amount of volume that will be dispensed. The contact area between the piston tip and the plate, i.e., the loading radius, is associated with maximum deflection and stresses developed. A bending formula for the plate is first developed and then used to solve for the bending line, the volume dispensed, and the actuation force required. The results are then used to design the membrane and to approximate the amount of force required to dispense a desired volume.

Droplet ejection modeling is also necessary to understand the influence of geometric and operating parameters associated with changes in flow inside the chamber. Therefore, a detailed ejection analysis was performed by solving for the fluid flow inside the chamber and the nozzle while considering surface tension, gravity, and free-surface change. A system representing a half section of one dispensing unit that consists of a chamber, an inlet port, and a nozzle is used for finite element modeling. Numerical studies were carried out to achieve a better understanding of the effects of geometric and operating variables on the device performance, and results were used to optimize the design of the dispensing device. Numerical tests were also used to examine droplet breakup, velocity, and volume by varying system parameters, including chamber diameter, chamber height, inlet length, inlet thickness, inlet location, and exit diameter of the nozzle.

\section{A. Static Analysis of the Plate}

When an electrical pulse is applied to the piezoactuator, the piston tip displaces a thin flexible plate and forces the liquid to move out from the nozzle. An analytical model that establishes a relationship between the loads applied to the plate and the volume of liquid dispensed is presented. Each pressure chamber consists of a through hole covered with a circular plate. The plate deformation is described by the elastic theory of a circular plate of radius $R$ and thickness $h$ with clamped edges [Fig. 2(a)]. A uniformly distributed load along the bottom of the plate characterizes the resistance of an incompressible fluid confined underneath the plate. The actuation load acts on a loading radius $R_{*}$ via the piston tips with intensity $\bar{q}_{1}$, and the fluid resistance has intensity $\bar{q}_{2}$. The radial coordinate of the circular plate and its lateral deflection are denoted as $\bar{r}$ and $\bar{w}$, respectively. Note that when the same letters are used to denote dimensional and their nondimensional quantities, an overbar above the dimensional values is added.

The plate geometry and loading conditions are symmetric about a central axis; therefore, the problem is treated axisymmetrically. The axisymmetric static deformation of a circular plate is described by [27], [28]

$$
\begin{aligned}
\frac{1}{\bar{r}} \frac{d}{d \bar{r}}\left\{\bar{r} \frac{d}{d \bar{r}}\left[\frac{1}{\bar{r}} \frac{d}{d \bar{r}}\left(\bar{r} \frac{d \bar{w}}{d \bar{r}}\right)\right]\right\}=\frac{\bar{q}}{D}, \\
\bar{q} \equiv \begin{cases}\bar{q}_{1}-\bar{q}_{2}, & 0 \leq \bar{r} \leq R_{*} \\
-\bar{q}_{2}, & R_{*}<\bar{r} \leq R\end{cases}
\end{aligned}
$$

where $D$ is the bending stiffness of the plate with Young's modulus $E$ and Poisson's ratio $\nu$. The bending stiffness is the product of the modulus of elasticity and the moment of inertia and is expressed as $D=E h^{3} /\left[12\left(1-\nu^{2}\right)\right]$ [27].

Equation (1) can be normalized to reduce parameters and to make it dimensionless by using the following parameters:

$$
\begin{aligned}
\bar{r} & =R r, \quad R_{*}=R r_{*}, \quad \bar{w}=h w, \quad \bar{q}=\bar{q}_{\diamond} q, \\
\bar{q}_{1} & =\bar{q}_{\circ} q_{1}, \quad \bar{q}_{2}=\bar{q}_{\circ} q_{2}, \quad \xi=h / R .
\end{aligned}
$$

The radial distance is normalized using the chamber outer radius $R$; the deflection is normalized using the thickness of the plate $h ; \xi$ is the ratio of the thickness of the plate to the radius of the plate. To simplify (1), pressure is normalized using a pressure scale $\bar{q}_{\circ}=h D / R^{4}=\xi^{4} E /\left[12\left(1-\nu^{2}\right)\right]$. Then, the normalized form of (1) is

$$
\begin{aligned}
\frac{1}{r} \frac{d}{d r}\left\{r \frac{d}{d r}\left[\frac{1}{r} \frac{d}{d r}\left(r \frac{d w}{d r}\right)\right]\right\} & =q, \\
q & \equiv \begin{cases}q_{1}-q_{2}, & 0 \leq r \leq r_{*} \\
-q_{2}, & r_{*}<r \leq 1\end{cases}
\end{aligned}
$$

New variables $w_{1}, w_{2}$, and $w_{3}$ are defined as

$$
w_{3} \equiv r\left(\frac{d w}{d r}\right), \quad w_{2} \equiv \frac{\left(d w_{3} / d r\right)}{r}, \quad w_{1} \equiv r\left(\frac{d w_{2}}{d r}\right)
$$

where $w_{3} / r=d w / d r$ is the first derivative of the bending line $w(r)$; therefore, $w_{3}$ is related to the slope of the bending line

$$
\begin{aligned}
w_{2} & =\frac{1}{r}\left(\frac{d w_{3}}{d r}\right)=\frac{d^{2} w}{d r^{2}}+\frac{1}{r} \frac{d w}{d r} \\
& =\frac{d^{2} w}{d r^{2}}+\frac{\nu}{r} \frac{d w}{d r}+\frac{1}{r} \frac{d w}{d r}-\frac{\nu}{r} \frac{d w}{d r} \\
& =M_{r r}+\frac{1-\nu}{r}\left(\frac{d w}{d r}\right)
\end{aligned}
$$




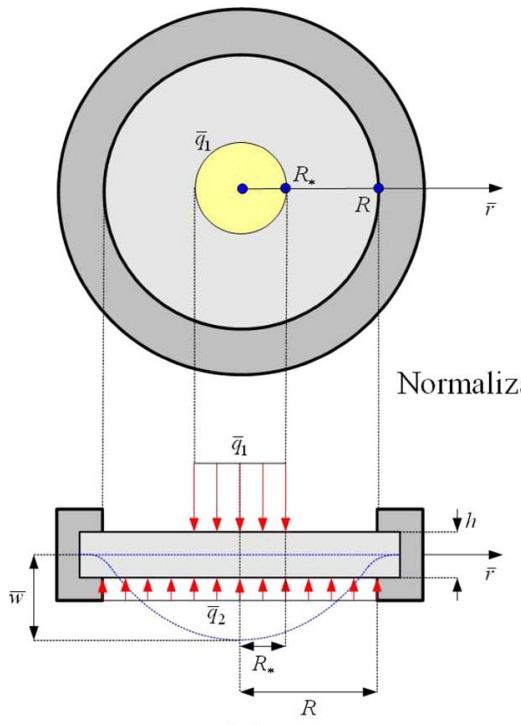

(a)

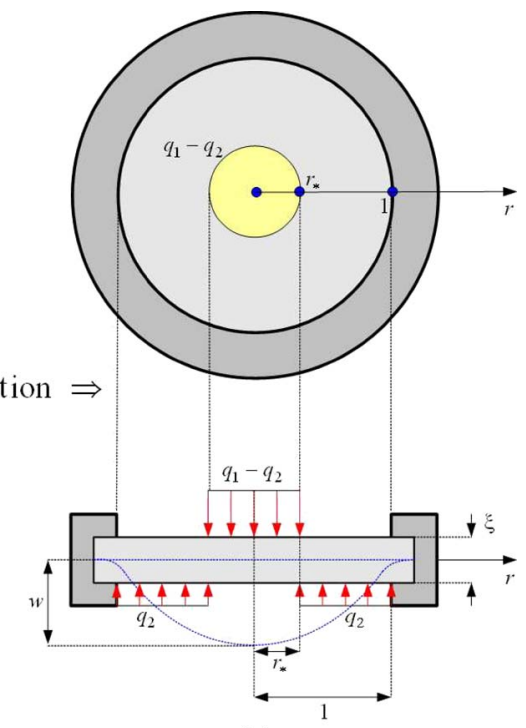

(b)

Fig. 2. Sketch of the loading condition of the circular plate with clamped edges. (a) Dimensional form. (b) Nondimensional form.

where $M_{r r}$ is the bending moment. Thus, $w_{2}$ relates to the combination of the bending moment and the slope

$$
w_{1}=r\left(\frac{d w_{2}}{d r}\right)=r \frac{d}{d r}\left[\frac{1}{r}\left(\frac{d}{d r}\left(r \frac{d w}{d r}\right)\right)\right]=-r Q_{r}
$$

where $Q_{r}$ represents the shear force. Therefore, $-\left(w_{1} / r\right)$ is the shear force.

Rewriting (3) using the variables $w_{1}, w_{2}$, and $w_{3}$

$$
\begin{aligned}
& \frac{d w_{1}}{d r}=r q, \quad q \equiv \begin{cases}q_{1}-q_{2}, & 0 \leq r \leq r_{*} \\
-q_{2}, & r_{*}<r \leq 1\end{cases} \\
& \frac{d w_{2}}{d r}=\frac{w_{1}}{r}, \quad \frac{d w_{3}}{d r}=r w_{2}, \quad \frac{d w}{d r}=\frac{w_{3}}{r} .
\end{aligned}
$$

The boundary conditions are the following:

1) zero slope at the center of the plate

$$
\left.\frac{d w}{d r}\right|_{r=0}=0
$$

2) zero slope at the clamped edges

$$
\left.\frac{d w}{d r}\right|_{r=1}=0
$$

3) no deflection at the clamped edges

$$
w(1)=0
$$

4) no concentrated force at the center of the plate [27]

$$
w_{1}(0)=0 .
$$

To illustrate the last boundary condition (6d), the relationship between the variable $w_{1}$ and the shear force $Q_{r}$ distributed on a circle with radius $r$ is [27]

$w_{1}=-r Q_{r},\left.\quad 2 \pi\left(r Q_{r}\right)\right|_{r=0}=-Q_{0} \quad \Rightarrow \quad w_{1}(0)=Q_{0} / 2 \pi$

where $Q_{0}$ is an externally applied concentrated force in the center of the plate. In this case, $Q_{0}$ is zero at the center of the plate because the force generated from the piezo is a distributed load.

The function $q$ in (5) is a step function and has two boundary regions (in terms of the radial parameter $r$ ). Therefore, to solve the system of (5) in the domain $0 \leq r \leq 1$, it is divided into two subdomains.

For the internal subdomain, $0 \leq r \leq r_{*}$

$$
\begin{aligned}
& \frac{d w_{1}^{(i)}}{d r}=r\left(q_{1}-q_{2}\right), \quad \frac{d w_{2}^{(i)}}{d r}=\frac{w_{1}^{(i)}}{r}, \\
& \frac{d w_{3}^{(i)}}{d r}=r w_{2}^{(i)}, \quad \frac{d w^{(i)}}{d r}=\frac{w_{3}^{(i)}}{r}, \\
& w_{1}^{(i)}(0)=0 ;\left.\quad \frac{d w^{(i)}}{d r}\right|_{r=0}=0 .
\end{aligned}
$$

For the external subdomain, $r_{*} \leq r \leq 1$

$$
\begin{aligned}
& \frac{d w_{1}^{(e)}}{d r}=-r q_{2}, \quad \frac{d w_{2}^{(e)}}{d r}=\frac{w_{1}^{(e)}}{r}, \frac{d w_{3}^{(e)}}{d r}=r w_{2}^{(e)}, \quad \frac{d w^{(e)}}{d r}=\frac{w_{3}^{(e)}}{r}, \\
& w^{(e)}(1)=0 ;\left.\quad \frac{d w^{(e)}}{d r}\right|_{r=1}=0 .
\end{aligned}
$$

Equations (8) and (9) are solved using the appropriate boundary conditions. At radius $r=r_{*}$, both (8) and (9) are matched 


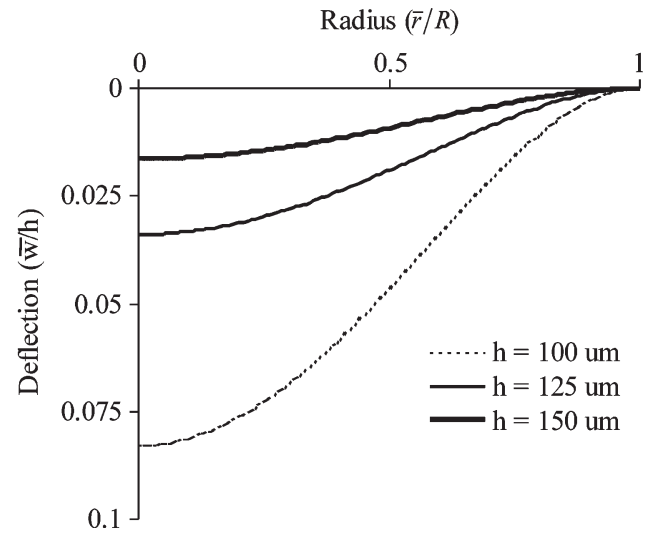

Fig. 3. Bending lines for different plate thickness.

to determine the plate lateral deflection in the two different subdomains as

$$
\begin{aligned}
w^{(i)}(r)=\frac{1}{64}\{ & {\left[q_{1} r_{*}^{2}\left(4-3 r_{*}^{2}+4 r_{*}^{2} \ln r_{*}\right)-q_{2}\right] } \\
& +2\left[q_{1} r_{*}^{2}\left(4 \ln r_{*}-r_{*}^{2}\right)+q_{2}\right] r^{2} \\
& \left.+\left(q_{1}-q_{2}\right) r^{4}\right\} \\
w^{(e)}(r)=\frac{1}{64}\{ & {\left[2 q_{1} r_{*}^{2}\left(2+r_{*}^{2}\right)-q_{2}\right] } \\
& -2\left[q_{1} r_{*}^{2}\left(2+r_{*}^{2}\right)-q_{2}\right] r^{2} \\
& \left.-q_{2} r^{4}+4 q_{1} r_{*}^{2}\left(r_{*}^{2}+2 r^{2}\right) \ln r\right\} .
\end{aligned}
$$

Fig. 3 shows the bending lines of the PDMS plates with a radius of $200 \mu \mathrm{m}$ for several plate thicknesses. The physical properties for a PDMS plate were described by Armani and Liu [29]. Results show that the maximum deflection for a $100-\mu \mathrm{m}-$ thick plate is $8.28 \mu \mathrm{m}$ (Fig. 3: $x$-axis $\bar{r} / R, y$-axis $\bar{w} / h$ ).

The amount of liquid ejected through the nozzle is the amount of liquid displaced by the deflection of the plate; thus, the dispensed volume is found from the integration of the bending line

$$
\begin{aligned}
V=2 \pi \int_{0}^{R} \bar{w} \bar{r} d \bar{r} & =2 \pi h R^{2}\left[v^{(i)}+v^{(e)}\right], \\
v^{(i)} & \equiv \int_{0}^{r_{*}} w^{(i)} r d r ; \quad v^{(e)} \equiv \int_{r_{*}}^{1} w^{(e)} r d r .
\end{aligned}
$$

Combining the solutions of (10) and (11) into (12) and integrating the two domains, the volume $V$ of the moving liquid is

$$
\begin{aligned}
V & =\frac{\pi\left(1-\nu^{2}\right) R^{6}\left[\bar{q}_{1} f\left(r_{*}\right)-\bar{q}_{2}\right]}{16 E h^{3}} \\
f\left(r_{*}\right) & \equiv r_{*}^{2}\left(r_{*}^{4}-3 r_{*}^{2}+3\right) .
\end{aligned}
$$

As we will see later in Section IV, the fluid resistance intensity $\bar{q}_{2}$ can be estimated from the experimental data. Therefore, the fluid resistance intensity is a known quantity.
To highlight the dependence of the dispensed volume on pressure, fluid resistance, and loading radius, (13a) is normalized as

$$
V=V_{\circ} v, \quad v=q_{1} f\left(r_{*}\right)-q_{2}
$$

where the scale for volume is $V_{\circ}=\pi \xi R^{3} / 192$.

Thus, for a given dispensed volume $v_{d}$, using (14), the intensity of the external load $q_{1}$ can be found as a function of the loading radius $r_{*}$ and the fluid resistance $q_{2}$

$$
q_{1}=\left(v_{d}+q_{2}\right) / f\left(r_{*}\right) .
$$

Equation (15) establishes the relationship between the volume dispensed and the actuation force. This equation provides a model to calculate the force required for dispensing a specified volume when the loading radius $r_{*}$ and the fluid resistance intensity $q_{2}$ are known.

The maximum deflection occurs at the center of the plate, and its value is found using (10) and (15) as

$$
w_{\max }\left(r_{*}\right)=\frac{1}{64}\left[\left(v_{d}+q_{2}\right) \frac{4-3 r_{*}^{2}+4 r_{*}^{2} \ln r_{*}}{r_{*}^{4}-3 r_{*}^{2}+3}-q_{2}\right] .
$$

From (13a) and (15), for a given dispensed volume $v_{d}$ and fluid resistance intensity $q_{2}$, all subsequent variables (bending line, maximum deflection, and actuation force) depend on the loading radius $r_{*}$. However, the loading radius $r_{*}$ itself is associated with the stresses developed in the system. Therefore, to select an appropriate value for the loading radius $r_{*}$, a stress analysis was performed. The geometric values used in producing the figures in the following analysis are for a PDMS circular plate with radius $R=200 \mu \mathrm{m}$ and thickness starting at $h=100 \mu \mathrm{m}$. Typical dimensions for the chamber diameter and the chamber height found in the literature range between 200 and $400 \mu \mathrm{m}$ and between 100 and $400 \mu \mathrm{m}$, respectively [8], [21], [22].

The stresses in the system can be solved to find the best value for the loading radius $r_{*}$, which results in a minimal value of the maximum stress across the entire plate.

The only nonzero components of the stress in this system are the radial $\bar{\sigma}_{r r}$ and tangential $\bar{\sigma}_{\theta \theta}$ stresses, which are as follows [27], [28]:

$$
\begin{array}{ll}
\bar{\sigma}_{r r}=\bar{\sigma}_{\circ} \sigma_{r r}, & \sigma_{r r}=\frac{d^{2} w}{d r^{2}}+\left(\frac{\nu}{r}\right) \frac{d w}{d r} \\
\bar{\sigma}_{\theta \theta}=\bar{\sigma}_{\circ} \sigma_{\theta \theta}, & \sigma_{\theta \theta}=\nu \frac{d^{2} w}{d r^{2}}+\left(\frac{1}{r}\right) \frac{d w}{d r}
\end{array}
$$

where $\bar{\sigma}_{\circ}=\xi^{2} E /\left[2\left(1-\nu^{2}\right)\right]$ is a normalizing coefficient for stress.

Nondimensional radial $\left(\sigma_{r r}\right)$ and tangential $\left(\sigma_{\theta \theta}\right)$ stresses for different loading radii $r_{*}$ are shown in Fig. 4, where it is found that both radial and tangential stresses are minimum and maximum at the center of the plate $(r=0)$ and at its edge $(r=1)$, respectively. Therefore, these stresses at the edges and the center are investigated through an optimization analysis and 


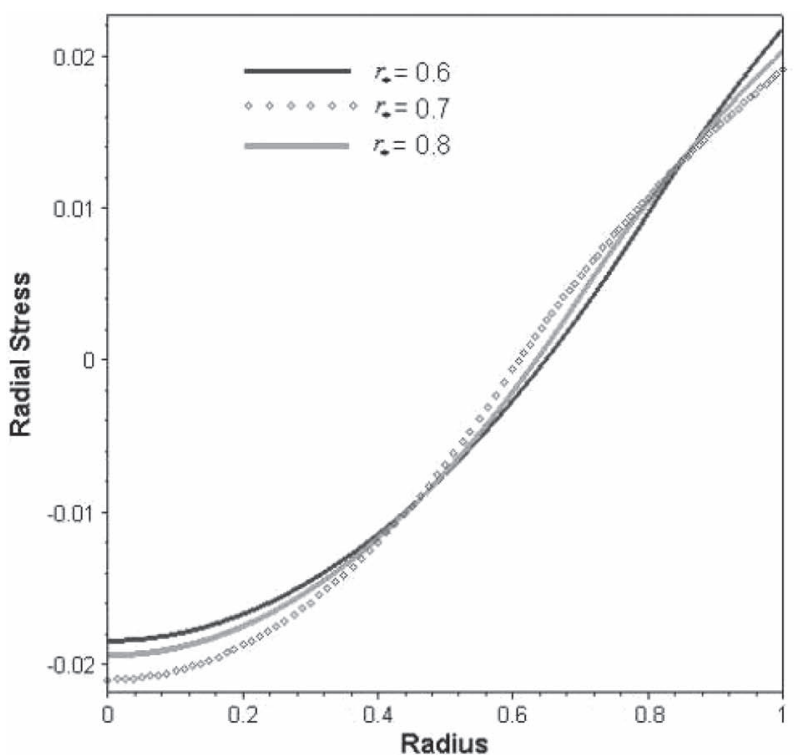

(a)

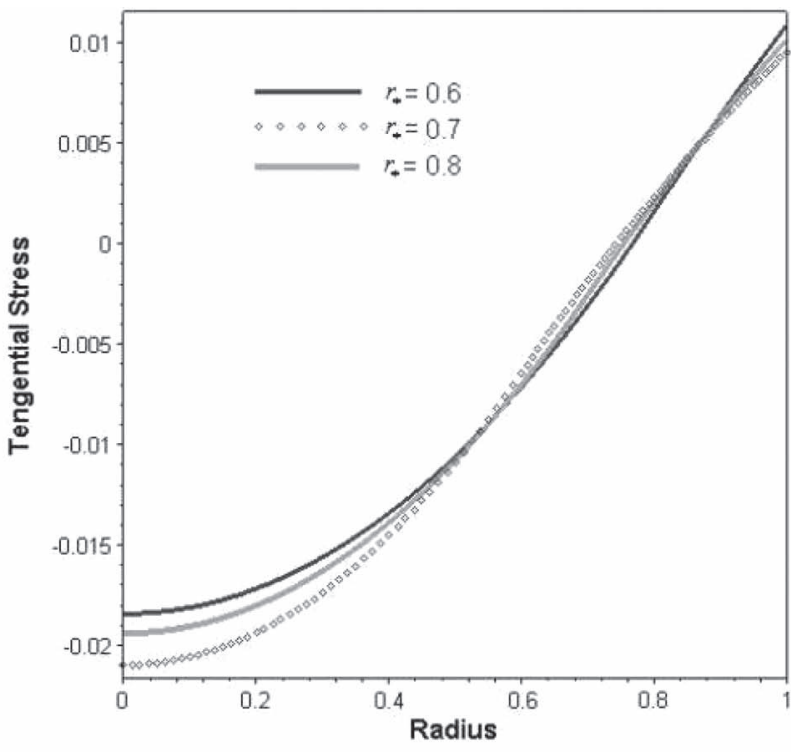

(b)

Fig. 4. Nondimensional (a) radial and (b) tangential stresses as a function of radius $r$.

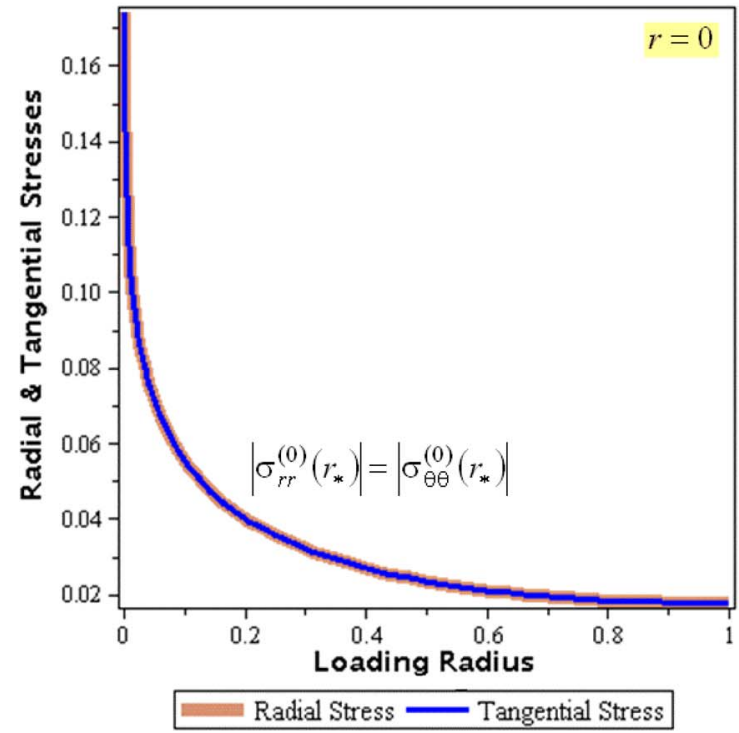

(a)

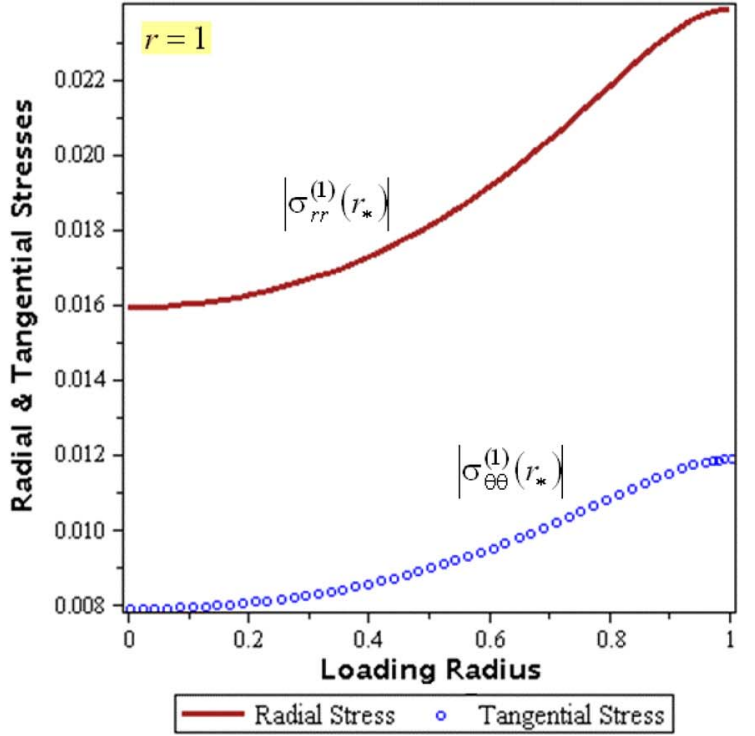

(b)

Fig. 5. Nondimensional radial and tangential stresses (a) in the plate's center and (b) at the plate's edge.

are compared for different loading radii. These two stresses are expressed in terms of the loading radius $r_{*}$

$$
\begin{aligned}
& \sigma_{r r}^{(0)}\left(r_{*}\right) \equiv\left[\sigma_{r r}(r)\right]_{r=0}, \quad \sigma_{\theta \theta}^{(0)}\left(r_{*}\right) \equiv\left[\sigma_{\theta \theta}(r)\right]_{r=0} \\
& \sigma_{r r}^{(1)}\left(r_{*}\right) \equiv\left[\sigma_{r r}(r)\right]_{r=1}, \quad \sigma_{\theta \theta}^{(1)}\left(r_{*}\right) \equiv\left[\sigma_{\theta \theta}(r)\right]_{r=1} .
\end{aligned}
$$

At $r=0,\left|\sigma_{r r}^{(0)}\left(r_{*}\right)\right|=\left|\sigma_{\theta \theta}^{(0)}\left(r_{*}\right)\right|$ [Fig. 5(a)]; thus, for design purposes, one could either consider the radial component or the tangential component at the center of the plate since both are equal. Similarly, Fig. 5(b) shows that $\left|\sigma_{\theta \theta}^{(1)}\left(r_{*}\right)\right|<\left|\sigma_{r r}^{(1)}\left(r_{*}\right)\right|$ and that the radial stress $\sigma_{r r}^{(1)}\left(r_{*}\right)$ is dominant. Therefore, two stress components are considered for design purposes: $\sigma_{r r}^{(0)}\left(r_{*}\right)$ and $\sigma_{r r}^{(1)}\left(r_{*}\right)$.
The best design value for the loading radius $r_{*}$ is where the maximum design stresses in the system [i.e., $\sigma_{r r}^{(1)}\left(r_{*}\right)$ and $\left.\sigma_{r r}^{(0)}\left(r_{*}\right)\right]$ are minimal. To find that value, the absolute values of the stress functions $\sigma_{r r}^{(0)}\left(r_{*}\right)$ and $\sigma_{r r}^{(1)}\left(r_{*}\right)$ are plotted in Fig. 6. The figure shows that these two stresses intersect at point $\mathrm{A}$ [Fig. 6(a)]. To the left of point A, the value of the radial stress developed at the center of the plate, i.e., $\sigma_{r r}^{(0)}\left(r_{*}\right)$, is higher compared to the other design stress, i.e., radial stress developed at the edge of the plate $\sigma_{r r}^{(1)}\left(r_{*}\right)$, and it increases gradually when going further to the left. To the right of point A, although $\sigma_{r r}^{(0)}\left(r_{*}\right)$ is decreasing, $\sigma_{r r}^{(1)}\left(r_{*}\right)$ gradually increases. Therefore, the maximal absolute value of the radial and tangential stresses across the whole plate is minimal at point A. From Fig. 6(b), the loading radius $\left(r_{*}^{\min }\right)$ at this intersection point is 0.664 . 


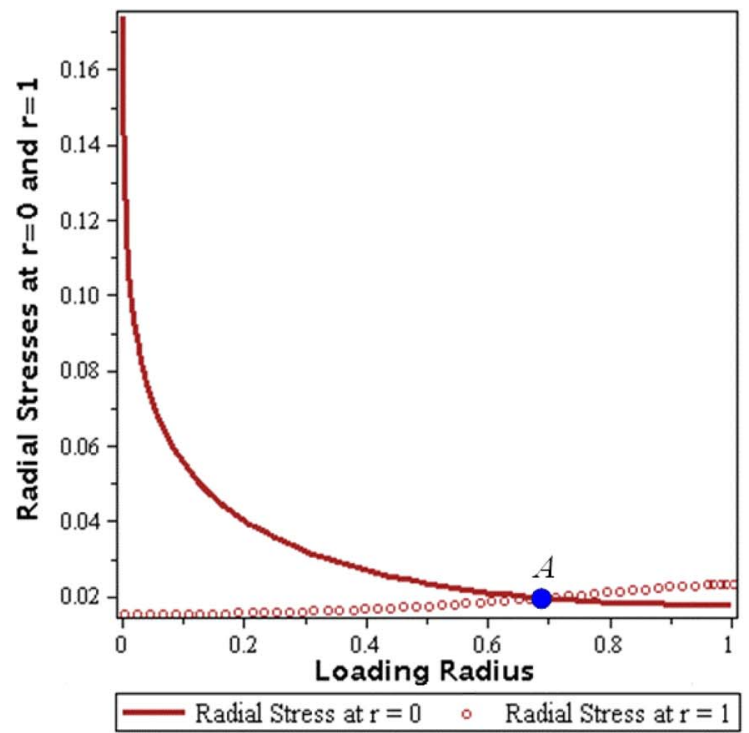

(a)

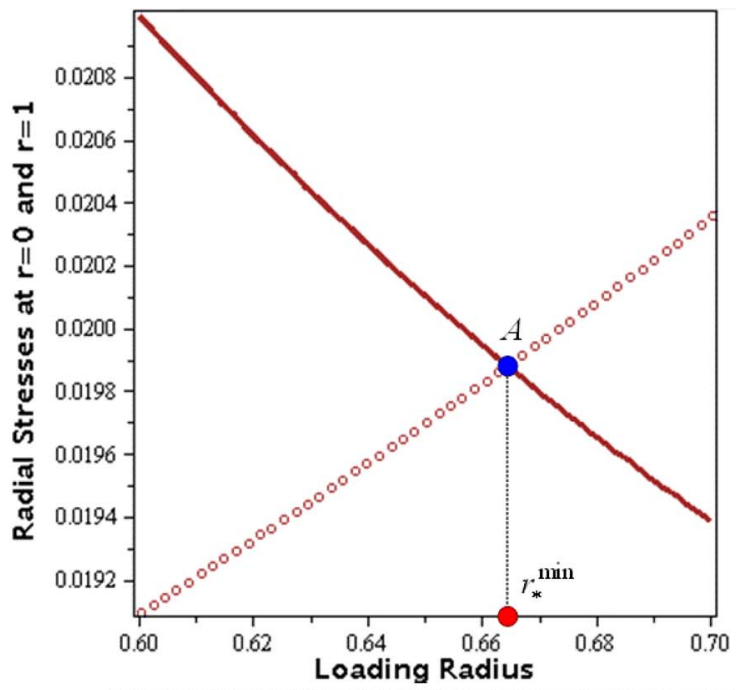

Radial Stress at $r=0 \quad \circ \quad$ Radial Stress at $r=1$

(b)

Fig. 6. (a) Optimal loading radius leads to a minimal value of the maximum stresses across the bending plate. (b) Enlarged view at the intersection of two stresses.

Therefore, this value corresponds to a loading radius where the values of the maximum stresses developed in the plate are minimum. This implies that the best size of the piston radius is $66.4 \%$ of the chamber radius.

\section{B. Modeling the Droplet Ejection Process}

The dispensing process was numerically modeled to allow parameter variations of design variables and to study device performance. ANSYS [30] was used to simulate the flow. The working fluid is incompressible and Newtonian. For the flow in microdroplet generators, surface forces are larger, therefore important, compared to volume forces because of the small device dimensions. The fluid domain moves with time, so the arbitrary Lagrangian-Eulerian algorithm was used [31]. Simulation of droplet formation involves tracking and solving the evolution of the free surface, at the tip of the nozzle; therefore, the volume of fluid (VOF) algorithm [32] was used. The aim was to find the most suitable values for the geometric and operating variables. Numerical experiments were carried out by varying the design parameters involved in the ejection process in order to examine their effect on the droplet generation.

The characteristic parameters analyzed were ejection sequence, droplet volume, dispensing frequency, droplet velocity, occurrence of satellite (unwanted) droplets, and velocity distribution inside the device. The following two groups of design parameters exist:

1) geometrical parameters: chamber diameter and height; nozzle diameter and height; and inlet length, height, and location;

2) operating parameters: driving pressure pulse type, frequency, and amplitude.

The numerical model considered the active region in the chip, i.e., the pressure chamber, the inlet, and the nozzle. Half the chip is used for the calculations to reduce the simulation time and the number of grid elements. A control volume represent-

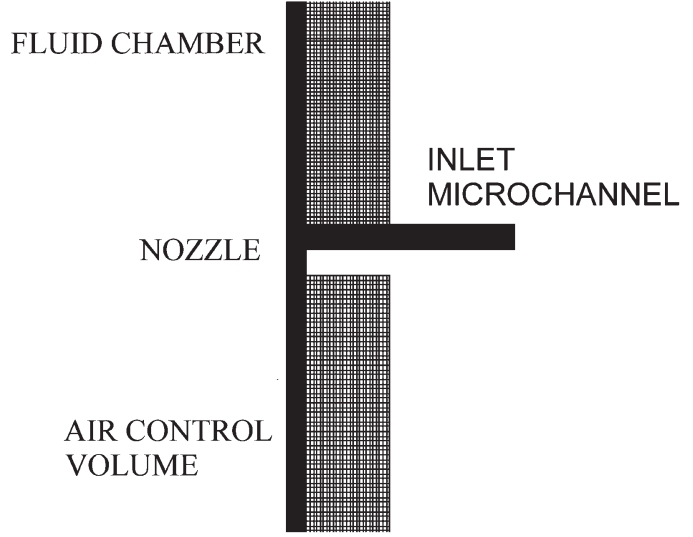

Fig. 7. Numerical domain and sample grid used in the numerical model. The numerical domain represents half of the dispensing device.

ing the surrounding air of the same dimension as the liquid pressure chamber was added beneath the nozzle to compute and visualize the droplet ejection sequences. The numerical domain shown in Fig. 7 represents half of the design shown in Fig. 1. The deformation of the plate was modeled in the previous section. Fluid flow in the chamber, nozzle, and inlet channel below the plate and droplet ejection sequence below the nozzle are modeled in this section. Water was used as the working fluid. A finer mesh was adopted in the regions (around the nozzle), as shown in Fig. 7, where the droplet creation and change in the free surface occur.

The boundary conditions are the following: The pressure pulse generated by the plate is applied at the top, the amplitude of which is calculated using (15). A constant low pressure was defined at the inlet, zero pressure at the nozzle exit, and all other surfaces as wall boundary conditions. It is a transient fluid flow problem involving both liquid and gas components.

Fig. 8 shows the ejection sequences from 0 to $0.8 \mathrm{~ms}$ in increments of $0.2 \mathrm{~ms}$. The evolution of the free surface from extrusion to droplet breakup gives a comprehensive image of 


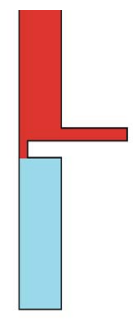

0 msec

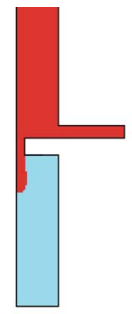

$0.2 \mathrm{msec}$

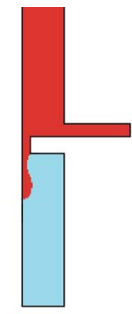

$0.4 \mathrm{msec}$

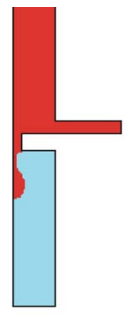

$0.6 \mathrm{msec}$

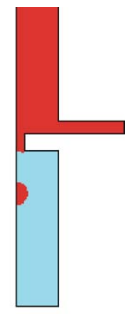

$0.8 \mathrm{msec}$

Fig. 8. VOF plots at different time instants showing droplet generation; red (dark) corresponds to liquid, and sky blue (light gray) corresponds to air.
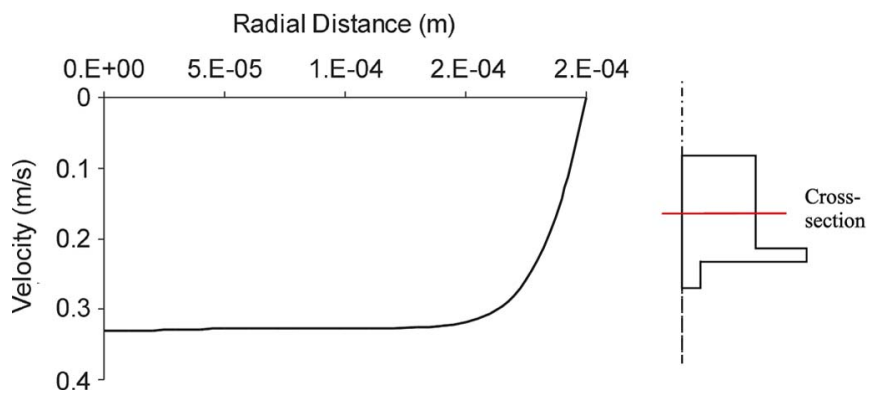

Fig. 9. Velocity profile inside the pressure chamber at the cross section shown to the right, at time $t=0.2 \mathrm{~ms}$.

the droplet dispensing. When the plate returns to the initial position, there is a negative pressure gradient inside the chamber that draws fluid into the chamber.

The plate deflects in one direction with one peak occurring at the center, which means that the velocity at the center of the chamber should be highest. The velocity profile at $0.2 \mathrm{~ms}$ is plotted in Fig. 9. It shows that the flow is developing inside the chamber with maximum velocity occurring at the center of the chamber. Velocity vectors at two time instants are shown in Fig. 10. Immediately after the actuation pulse, the constant low inlet pressure prevents liquid backflow. The velocity gradually decreases to zero inside the inlet channel, preventing liquid backflow [Fig. 10(a)]. When the plate returns to its initial position, the negative pressure pulse forces liquid to flow from the inlet to the chamber [Fig. 10(b)]. Thus, the refilling of the chip is initiated by the release of the plate that causes a negative pressure at the top of the pressure chamber, and the process is aided by the small overpressure maintained at the inlet.

The nozzle diameter directly affects droplet volume and velocity. The nozzle diameter was varied in the numerical runs and is linearly related to the droplet volume. The diameter is varied between 25 and $150 \mu \mathrm{m}$. Once the diameter exceeds $150 \mu \mathrm{m}$, nonuniform droplets are formed, and fluid comes out in the form of jets and then breaks into a main droplet and satellite droplets. Similarly, there is also a second value of $25 \mu \mathrm{m}$, below which no droplets could be generated because of high surface tension forces. Results also show that a longer and thinner inlet, as expected, leads to greater pressure loss. Moreover, it takes more time to fill the chambers from the reservoir if a longer inlet microchannel is used. If the inlet channel is too thin $(<5 \mu \mathrm{m})$, then the pressure loss will be large, and high power, as well as longer time, is necessary to refill the chambers. If the inlet channel height is too large $(>75 \mu \mathrm{m})$, no droplets are created, and fluid tends to move

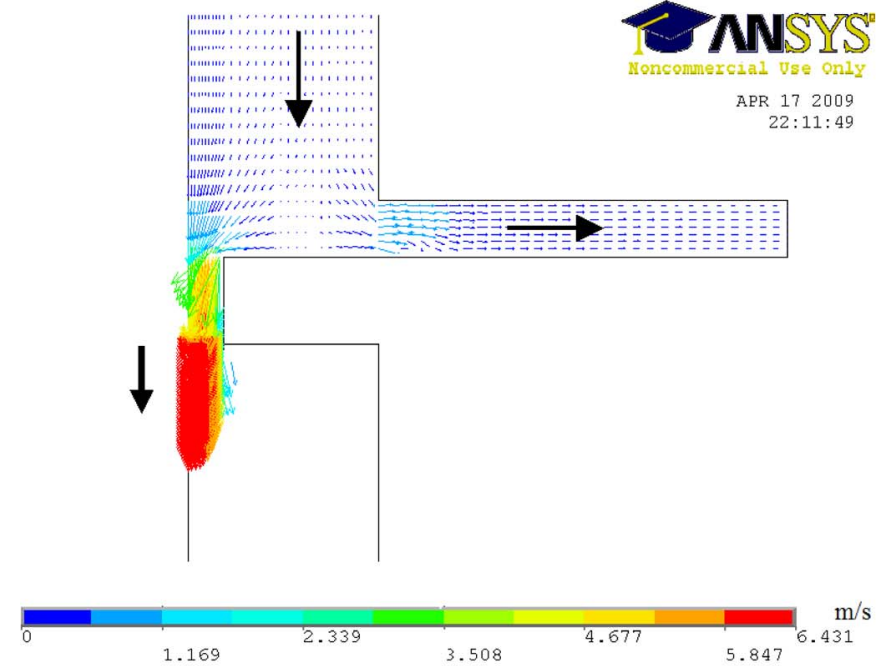

(a)

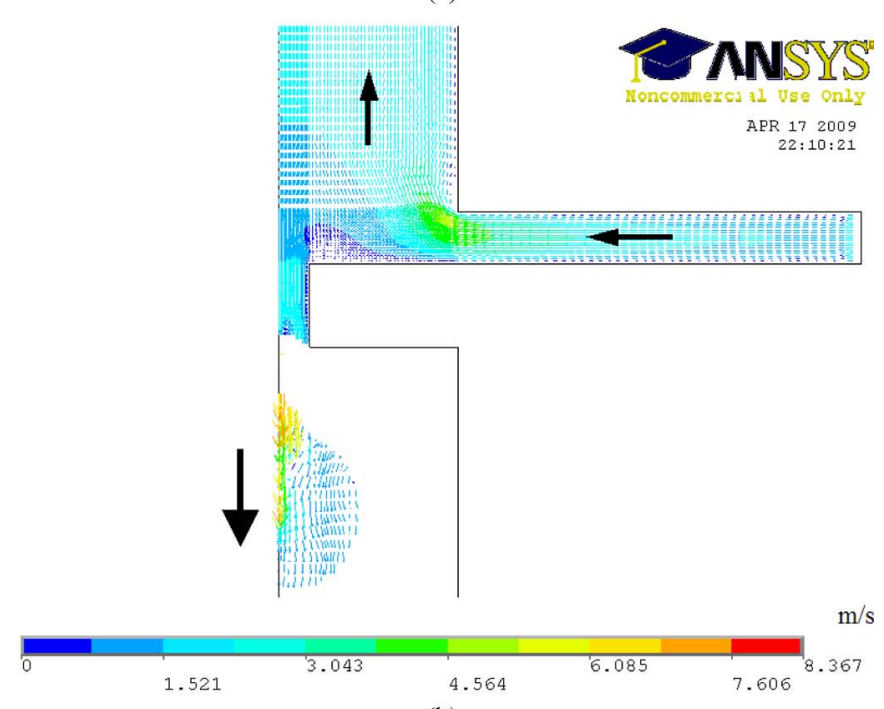

(b)

Fig. 10. Velocity vector distribution analysis at two time instants (beginning of droplet ejection and after droplet ejection): (a) Starting of fluid column ejection and (b) after droplet ejection. Big black arrows are placed on the ANSYS plots to show the flow direction.

into the inlet channel due to the lower pressure drop required for fluid to move to the inlet region as compared to the nozzle region.

Two inlet locations were considered for the design and were assessed numerically: 1) fluid is fed into the dispensing chamber from the top of the chamber or 2) from the bottom of the chamber. The average fluid velocity inside the chamber and 


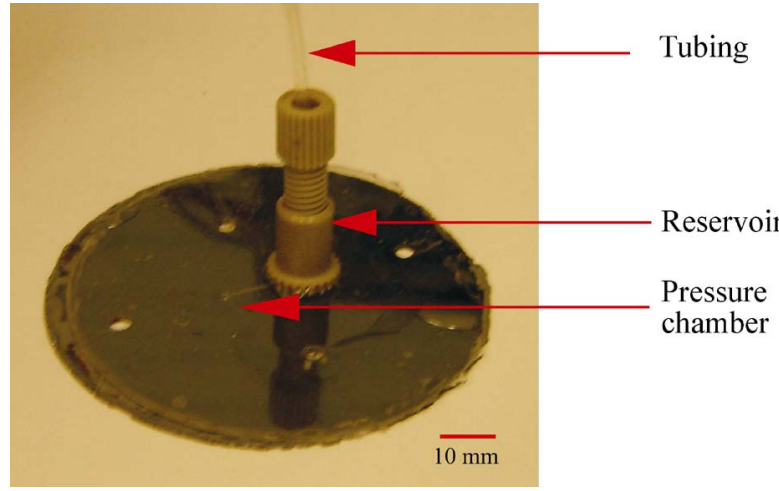

Fig. 11. Assembled prototype.

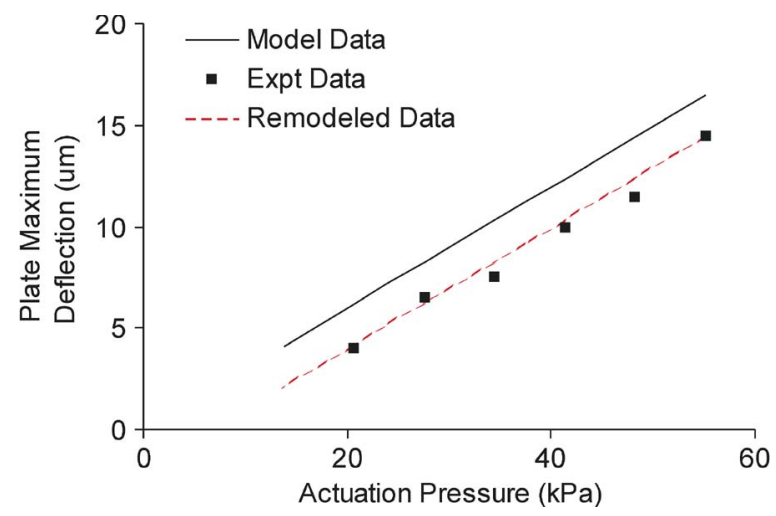

Fig. 12. Comparison between the analytical estimates and measurements of the maximum plate deflection corresponding to different actuation force intensities.

nozzle was approximately twice as large when the inlet was at the bottom. Thus, when the inlet is positioned at the bottom, it improved the flow, which improves the refilling process. The numerical simulations also show that, when the diameter of the dispensing chamber was too large $(>600 \mu \mathrm{m})$, it was not possible to dispense liquid.

\section{EXPERIMENTS}

A single dispensing unit was fabricated and tested. It consists of one chamber (400- $\mu \mathrm{m}$ diameter and $280-\mu \mathrm{m}$ height), one nozzle $(100-\mu \mathrm{m}$ diameter and $100-\mu \mathrm{m}$ height), and a microchannel (60 $\mu \mathrm{m}$ deep). The prototype is shown in Fig. 11. To validate the analytical model, the actuation force from the piezo and the maximum deflection of the membrane were measured. The plate model was evaluated by comparing the predicted maximum deflection with the measured data. The maximum deflections predicted from the model and experimental measurements are shown in Fig. 12, where the actuation force intensity is plotted against the maximum deflection (the solid line represents the case where $\bar{q}_{2}=0$ ). The force from the piezo was measured with a Kistler load washer force sensor 9001A. The maximum plate deflection was measured by recording the maximum displacement of the edge of the piezoactuator when it was attached to the piston tip by image analysis. An initial comparison between the model and experimental data shown in Fig. 12 reveals a good agreement on the slope. The model data at the initial comparison have a slope of 0.25 , whereas the experimental data have a slope of 0.28 . However, the model overpredicts the maximum deflection. To reduce the difference, a remodeling was necessary. In the development of the model, the pressure from the liquid $\bar{q}_{2}$ was assumed to equal to zero. The model was extended to solve for the pressure $\bar{q}_{2}$. Equation (10) solves for the deflection of the plate and could be used to calculate the maximum deflection at the center of the plate which can be expressed as

$$
\begin{aligned}
w_{\max }=w^{(i)}(0)=\frac{1}{64}\left(a_{*} q_{1}-q_{2}\right), & \\
& a_{*} \equiv r_{*}^{2}\left(4-3 r_{*}^{2}+4 r_{*}^{2} \ln r_{*}\right) .
\end{aligned}
$$

From the aforementioned equation, the maximum deflection $w_{\max }$ is a linear function of the applied load $q_{1}$. This finding agrees with the experimental data shown in Fig. 12.

Equation (21) is an expression for a straight line, where the parameter $q_{2}$ (pressure from the liquid underneath the membrane) is the $y$ intercept and is responsible for moving the line in the $y$-direction. We can calculate $q_{2}$ from (21) using the available experimental data for $w_{\max }$ and $q_{1}$. Rearranging (21) leads to

$$
q_{2}=a_{*} q_{1}-64 w_{\max } .
$$

Using the data for the applied pressure load $q_{1}$ and the maximum deflection $w_{\max }\left\{\widetilde{q}_{1}^{(k)}, \widetilde{w}_{\max }^{(k)} ; k=1, \ldots, N\right\}$, an estimate of the fluid pressure $q_{2}$ using the least-squares method is obtained

$$
\hat{q}_{2}=\frac{1}{N} \sum_{k=1}^{N}\left[a_{*} \widetilde{q}_{1}^{(k)}-64 \widetilde{w}_{\max }^{(k)}\right] .
$$

For the six experimental points in Fig. 12, $N=6$. Once the value of the fluid resistance is estimated, its value was used in the model to recalculate the theoretical deflection, and results are shown in Fig. 12 (dashed line). The data obtained using the improved approach are in good agreement with the experimental data.

A microscope mounted with a high-speed camera recorded the ejection of droplets. Another image capturing device (courtesy of the Canadian Microelectronics Corporation) was also placed close to the device to study repeatability. Several snapshots showing the droplet ejection sequence at the tip of the nozzle at different time intervals after the actuation of the piezo are shown in Fig. 13, along with numerical predictions. These images were used to compare results from numerical modeling. Five consecutive droplets were dispensed for each experiment. The deviation between subsequent droplet volumes was less than $10 \%$. The changes in free-surface shape captured in the numerical simulation and experimentally are compared in Fig. 13, and similar breakup characteristics with no occurrence of unwanted (satellite) droplet formation were found. There was a small dead volume remaining at the nozzle tip after ejection, which is approximately twice the diameter of the nozzle [Fig. 13(a) and (f)]. Experiment results showed that this volume did not alter the repeatability, as it remained the same during the subsequent ejections. However, it limits the 


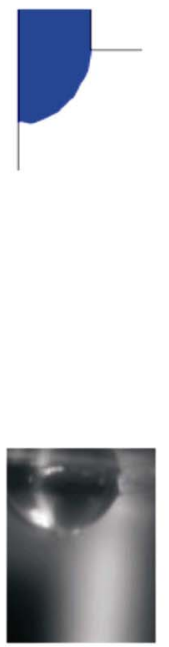

(a)
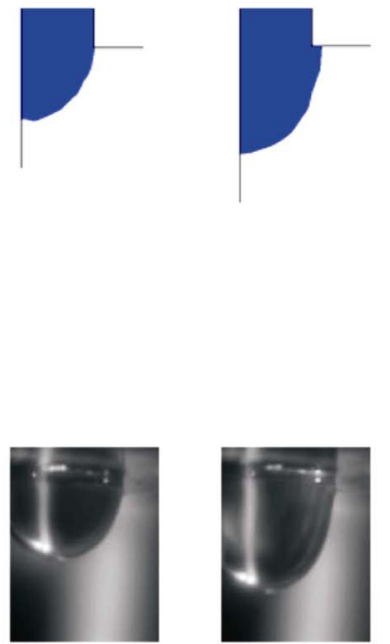

(b)

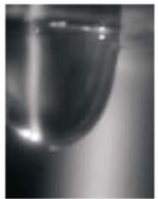

(c)
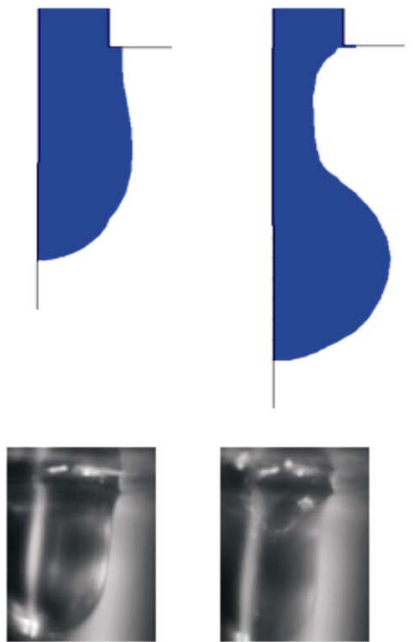

(d)

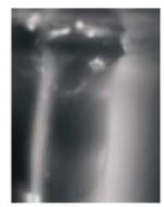

(e)
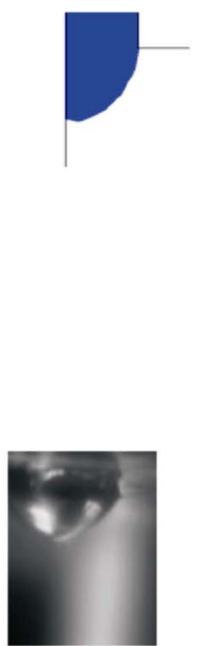

(f)

Fig. 13. (Top) Numerical predictions with (bottom) experimentally visualized images showing sequences of droplet breakup at different time instants: (a) $0.03 \mathrm{~s}$; (b) $0.06 \mathrm{~s}$; (c) $0.08 \mathrm{~s}$; (d) $0.1 \mathrm{~s}$; (e) $0.12 \mathrm{~s}$; and (f) $0.14 \mathrm{~s}$.

separation distance between two subsequent nozzles to half of the diameter to avoid cross contamination between neighboring nozzles.

The volume of the droplets ejected from the prototype was $1 \mathrm{~nL}$, while in the simulations with similar geometric parameters, it was $600 \mathrm{pL}$. The difference between numerical and experimental volumes is likely due to the influence of the dead volume and inaccuracy in the fabrication process such as the nozzle size which was found to be $15 \%-20 \%$ higher in the prototype.

\section{CONCLUSION}

A compact mathematical model based on mechanical buckling of a plate-actuated fluidic dispensing system was developed that describes the bending characteristics of the plate. The model is useful in predicting the actuation force required to dispense a desired volume. This actuation force was used as input to a dispenser numerical model and is used for selecting the actuator characteristics. The bending formula and force calculations were also used to design the plate physical properties. The model was then extended to perform stress analysis to determine the optimum loading radius of the actuating force. The model showed that the loading radius should be $\sim 67 \%$ of the chamber radius to minimize stress in the system. The droplet ejection process was simulated numerically in order to design the overall dispensing device. Numerical runs led to critical values for the geometric and operating variables. It showed that a minimum droplet volume of $50 \mathrm{pL}$ is possible at a maximum dispensing frequency of $6 \mathrm{kHz}$ without forming satellite droplets. The fabricated device showed repeatable dispensing without creating any satellite droplets. The good agreement between the model predictions and the experimental results demonstrates the usefulness of these models as a design tool.

\section{ACKNOWLEDGMENT}

The authors would like to thank M. Lundberg (KTH, Sweden) for his contributions to the early stages of the project.

\section{REFERENCES}

[1] M. Schena, Microarray Biochip Technology. Westborough, MA: Eaton, 2000.

[2] E. Zubritsky, "Product review: Spotting a microarray system," Anal. Chem., vol. 72, no. 23, pp. 761A-767A, Dec. 1, 2000.

[3] I. Barbulovic-Nad, M. Lucente, Y. Sun, M. Zhang, A. R. Wheeler, and M. Bussmann, "Bio-microarray fabrication techniques-A review," Crit. Rev. Biotech., vol. 26, no. 4, pp. 237-259, Dec. 2006.

[4] L. Jae-Duk, Y. Jun-Bo, K. Jae-Kwan, C. Hoon-Ju, L. Choon-Sup, L. Hi-Deok, L. Ho-Jun, K. Choong-Ki, and H. Chul-Hi, "A thermal inkjet printhead with a monolithically fabricated nozzle plate and self-aligned ink feed hole," J. Microelectromech. Syst., vol. 8, no. 3, pp. 229-236, Sep. 1999.

[5] T. Fan-Gang, K. Chang-Jin, and H. Chih-Ming, "A high-resolution high-frequency monolithic top-shooting microinjector free of satellite drops-Part II: Fabrication, implementation, and characterization," J. Microelectromech. Syst., vol. 11, no. 5, pp. 437-447, Oct. 2002.

[6] A. K. Sen and J. Darabi, "Droplet ejection performance of a monolithic thermal inkjet print head," J. Micromech. Microeng., vol. 17, no. 8, pp. 1420-1427, Aug. 2007.

[7] J. M. Wissink and C. J. M. Van Rijn, "Smart micromachined nozzles for monodisperse aerosol generation using low pressure Rayleigh break-up," in Proc. Respiratory Drug Delivery VIII, 2002, vol. 2, pp. 509-512.

[8] P. Koltay, R. Steger, B. Bohl, and R. Zengerle, "The dispensing well plate: A novel nanodispenser for the multiparallel delivery of liquids (DWP Part I)," Sens. Actuators A, Phys., vol. 116, no. 3, pp. 483-491, Oct. 2004.

[9] W. K. Choi, E. Lebrasseur, M. I. Al-Haq, H. Tsuchiya, T. Torii, H. Yamazaki, E. Shinohara, and T. Higuchi, "Nano-liter size droplet dispenser using electrostatic manipulation technique," Sens. Actuators A, Phys., vol. 136, no. 1, pp. 484-490, May 2007.

[10] F. Takagi, R. Kurosawa, D. Sawaki, and S. Kamisuki, "Picoliter dispenser with 128 independent nozzles for high throughput biochip fabrication," in Proc. 17th Int. Conf. Micro Electro Mech. Syst., Maastricht, The Netherlands, 2004, pp. 276-279.

[11] U. Demirci, "Acoustic picoliter droplets for emerging applications in semiconductor industry and biotechnology," J. Microelectromech. Syst., vol. 15, no. 4, pp. 957-966, Aug. 2006.

[12] D. Huang and E. S. Kim, "Micromachined acoustic-wave liquid ejector," J. Microelectromech. Syst., vol. 10, no. 3, pp. 442-449, Sep. 2001.

[13] H. B. Hsieh, J. Fitch, D. White, F. Torres, J. Roy, R. Matusiak, B. Krivacic, B. Kowalski, R. Bruce, and S. Elrod, "Ultra-high-throughput microarray generation and liquid dispensing using multiple disposable piezoelectric ejectors," J. Biomol. Screen., vol. 9, no. 2, pp. 85-94, Mar. 2004.

[14] A. Yang, J. Yang, and M. Hong, "Droplet ejection study of a PicoJet printhead," J. Micromech. Microeng., vol. 16, no. 1, pp. 180-188, Jan. 2006

[15] B. de Heij, C. Steinert, H. Sandmaier, and R. Zengerle, "A tuneable and highly-parallel picolitre-dispenser based on direct liquid displacement," Sens. Actuators A, Phys., vol. 103, no. 1/2, pp. 88-92, Jan. 2003. 
[16] C. P. Steinert, I. Goutier, O. Gutmann, H. Sandmaier, M. Daub, B. de Heij, and R. Zengerle, "A highly parallel picoliter dispenser with an integrated, novel capillary channel structure," Sens. Actuators A, Phys., vol. 116, no. 1, pp. 171-177, Oct. 2004.

[17] J. Ducree, H. Gruhler, N. Hey, M. Muller, S. Bekesi, M. Freygang, H. Sandmaier, and R. ZengerleIp, "Topspot-A new method for the fabrication of microarrays," in Proc. 13th Int. Conf. Micro Electro Mech. Syst., Miyazaki, Japan, 2000, pp. 317-322.

[18] S. B. Fuller, E. J. Wilhelm, and J. M. Jacobson, "Ink-jet printed nanoparticle microelectromechanical systems," J. Microelectromech. Syst., vol. 11, no. 1, pp. 54-60, Feb. 2002.

[19] J. Bergkvist, T. Lilliehorn, J. Nilsson, S. Johansson, and T. Laurell, "Miniaturized flow through microdispenser with piezoceramic tripod actuation," J. Microelectromech. Syst., vol. 14, no. 1, pp. 134-140, Feb. 2005

[20] C. H. Cheng, A. S. Yang, and T. H. Hsu, "Processing of PZT actuator and nickel plate in a multi-droplets microejector," J. Mater. Process. Technol., vol. 201, no. 1-3, pp. 683-688, May 2008.

[21] Y. S. Chen, Y. L. Huang, C. H. Kuo, and S. H. Chang, "Investigation of design parameters for droplet generators driven by piezoelectric actuators," Int. J. Mech. Sci., vol. 49, no. 6, pp. 733-740, Jun. 2007.

[22] P. Koltay, J. Kalix, and R. Zengerle, "Theoretical evaluation of the dispensing well plate method (DWP Part II)," Sens. Actuators A, Phys., vol. 116, no. 3, pp. 472-482, Oct. 2004.

[23] C. D. Meinhart and H. Zhang, "The flow structure inside a microfabricated inkjet printhead," J. Microelectromech. Syst., vol. 9, no. 1, pp. 67-75, Mar. 2000.

[24] P. Ben-Tzvi, R. Ben-Mrad, and A. A. Goldenberg, "A conceptual design and $\mathrm{FE}$ analysis of a piezoceramic actuated dispensing system for microdrops generation in microarray applications," Mechatronics, vol. 17, no. 1, pp. 1-13, Feb. 2007.

[25] T. Fan-Gang, K. Chang-Jin, and H. Chih-Ming, "A high-resolution high-frequency monolithic top-shooting microinjector free of satellite drops-Part I: Concept, design, and model," J. Microelectromech. Syst., vol. 11, no. 5, pp. 427-436, Oct. 2002.

[26] M. Nakamura, A. Kobayashi, F. Takagi, A. Watanabe, Y. Hiruma, K. Ohuchi, Y. Iwasaki, M. Horie, I. Morita, and S. Takatani, "Biocompatible inkjet printing technique for designed seeding of individual living cells," Tissue Eng., vol. 11, no. 11/12, pp. 1658-1666, Nov. 2005.

[27] J. N. Reddy, Theory and Analysis of Elastic Plates. Philadelphia, PA: Taylor \& Francis, 1999.

[28] S. Timoshenko and S. Woinowsky-Krienger, Theory of Plates and Shells, 2nd ed. New York: McGraw-Hill, 1987.

[29] D. Armani and C. Liu, "Re-configurable fluid circuits by PDMS elastomer micromachining," in Proc. 12th Int. Conf. Micro Electro Mech. Syst., Orlando, FL, 1999, pp. 222-227.

[30] ANSYS User Manual, ANSYS, Inc., Canonsburg, PA. [Online]. Available: http://www.ansys.com

[31] C. W. Hirt, A. A. Amsden, and J. L. Cook, "An arbitrary Lagrangian-Eulerian computing method for all flow speeds," J. Comput. Phys., vol. 135, no. 2, pp. 203-216, Aug. 1997.

[32] C. W. Hirt and B. D. Nichols, "Volume of fluid (VOF) method for the dynamics of free boundaries," J. Comput. Phys., vol. 39, no. 1, pp. 201225, Jan. 1981.

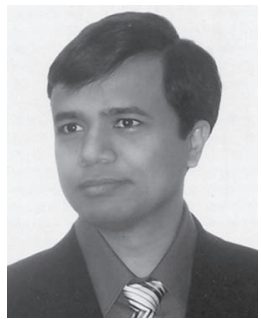

Mohammed Jalal Ahamed received the B.Sc. and M.Sc. degrees in mechanical engineering from Bangladesh University of Engineering and Technology (BUET), Dhaka, Bangladesh, in 2002 and 2004, respectively, and the M.A.Sc. degree in mechanical engineering from the University of Toronto, Toronto, ON, Canada, in 2006, where he is currently working toward the Ph.D. degree.

His research interests include MEMS, microfluidic systems, and lab-on-a-chip technology.

Mr. Ahamed was the recipient of the Dr. V. G. Desa Gold Medal from BUET and the Connaught Fellowship at the University of Toronto.

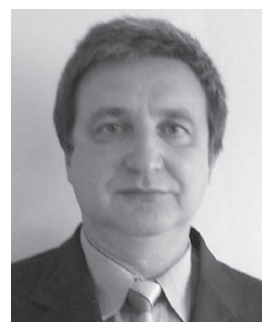

Sergey I. Gubarenko received the M.Sc. degree in applied mechanics from Moscow State University, Moscow, Russia, in 1977, and the Ph.D. and D.Sc. degrees in theoretical and applied mechanics from Moscow Power Engineering Institute, Moscow, Russia, in 1980 and 1994, respectively.

From 1986 to 2003, he was a Professor and the Deputy Manager of the Department of Theoretical Mechanics and Mechatronics, Moscow Power Engineering Institute, Moscow, Russia. Since 2004, he has been with the research staff of the Department of Mechanical and Industrial Engineering, University of Toronto, Toronto, ON, Canada. He is currently engaged in the research fields of electrohydrodynamics, digital microfluidics, fluid-structure interactions, perturbation methods in applied mechanics, inverse problems in elasticity, and energy harvesting. He is involved in R\&D projects focused on electrospray emitters, microdispensers, micropumps, motion of droplets on dielectrics, microelectromechanical gyros, damage detection in the auto industry, and piezoelectric transducers in energy harvesting microdevices.

Dr. Gubarenko is a member of the Navigation and Motion Control Academy, Saint Petersburg, Russia.

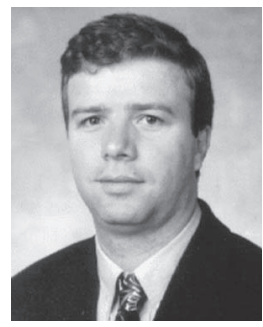

Ridha Ben-Mrad received the B.Sc. degree in mechanical engineering from The Pennsylvania State University, University Park, in 1986, and the M.A.Sc. degree in mechanical engineering, the M.Sc. degree in electrical engineering, and the Ph.D. degree in mechanical engineering from the University of Michigan, Ann Arbor, in 1988, 1991, and 1994, respectively.

Since 1997, he has been with the Department of Mechanical and Industrial Engineering, University of Toronto, Toronto, ON, Canada, where he is currently a Professor. His research interests include MEMS fabrication and design, microfluidics, design of piezoceramic-actuated linear motors, and development and characterization of smart-material-based sensors and actuators.

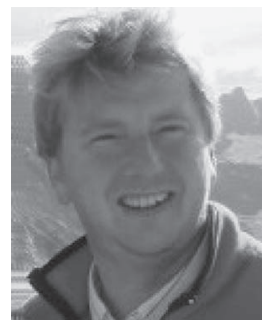

Pierre Sullivan received the Ph.D. degree from Queen's University, Kingston, ON, Canada, in 1995.

$\mathrm{He}$ is currently with the Department of Mechanical and Industrial Engineering, University of Toronto, Toronto, ON. His research interests span turbulent flow phenomena, including aerodynamic control for microair vehicles, energy conservation, and microscale electrohydrodynamics. He has focused on the development of innovative experimental techniques for these systems with associated modeling. 